A. V. Medvedev * Department of Mechanics and Mathematics, Moscow State University, Moscow, Russia. e-mail: andrey@medvedev.mccme.ru

\title{
ON A CONCAVE DIFFERENTIABLE MAJORANT OF A MODULUS OF CONTINUITY
}

\begin{abstract}
In this paper we prove that for any modulus of continuity on $[0, \infty)$ there exists a concave majorant that is infinitely differentiable on $(0, \infty)$ and satisfies an additional inequality. This extends the results of Stechkin and Korneychuk obtained previously without the requirement that majorants be differentiable.
\end{abstract}

Recall that a real function $\omega$ on $[0, \infty)$ or on $[0, l], 0<l<\infty$, is called a modulus of continuity if $\omega$ is continuous, semiadditive, nondecreasing and $\omega(0)=0$. The concavity of $\omega$ is sometimes a desirable property but in general $\omega$ fails to be concave. In certain cases this difficulty can be surmounted by using a concave majorant of $\omega$. Throughout this paper we assume that $\omega$ differs from the zero function. The following lemma is due to S. B. Stechkin. It was published and applied for the first time in [1].

Lemma A. Let $\omega$ be a modulus of continuity on $[0, \pi]$. Then there exists a concave modulus of continuity $\bar{\omega}$ such that $\omega(t) \leq \bar{\omega}(t)<2 \omega(t)$ for $t \in(0, \pi]$. Moreover, the constant 2 cannot be reduced.

The proof in [1] remains valid if $\pi$ is replaced by any positive number $l$. Later N. P. Korneychuk [2] proved the following lemma.

Lemma B. Let $\omega$ be a modulus of continuity on $[0, \infty)$ and $\bar{\omega}$ be the minimal concave majorant of $\omega$. Then $\bar{\omega}(\mu t)<(1+\mu) \omega(t)$ for any $t>0, \mu>0$. This inequality is best possible for each $t>0$ and each natural $\mu$.

\footnotetext{
Key Words: modulus of continuity, majorant, concave

Mathematical Reviews subject classification: 26A15

Received by the editors October 2, 2000

* This research was carried out with the partial financial support of the Russian Foundation for Basic Research (grant no.99-01-00062) and the program "Leading Scientific Schools" (grant no.00-15-96143).
} 
The construction of $\bar{\omega}$ in $[1,2]$ proves that any modulus of continuity has a minimal concave majorant which is a modulus of continuity as well. As is known any concave function of one variable is differentiable at each point in its domain except at most a countable set. The question that we are dealing with was suggested by P. L. Ul'janov. Let $\omega$ be a modulus of continuity on $[0, \infty)$. The question is whether there exist a constant $c$ and a concave modulus of continuity $\omega_{0}$ on $[0, \infty)$ so that the restriction of $\omega_{0}$ to $(0, \infty)$ has a given order of smoothness and satisfies $\omega(t) \leq \omega_{0}(t)<c \omega(t)$. It is natural in view of Lemma $\mathrm{B}$ to consider the problem with the inequality of the form $\omega_{0}(\mu t)<c(\mu) \omega(t)$. The next theorem yields an answer to Ul'janov's problem.

Theorem. Let $\omega$ be a modulus of continuity on $[0, \infty)$ and $I$ be a closed interval in $(0, \infty)$. Then there exists a concave modulus of continuity $\omega_{0}$ on $[0, \infty)$ such that the restriction of $\omega_{0}$ to $(0, \infty)$ is infinitely differentiable and satisfies $\omega(\mu t) \leq \omega_{0}(\mu t)<(1+\mu) \omega(t)$ for $t>0$ and $\mu \in I$. Moreover, if $\omega^{\prime}(0)<\infty$, then $\omega_{0}(t)=\omega^{\prime}(0) t$ on some neighborhood of zero.

To prove the theorem we need two more lemmas. In Lemma 1 it is possible that $\bar{\omega}^{\prime}(0)=\infty$.

Lemma 1. Let $\omega$ be a modulus of continuity on $[0, \infty)$ and $\bar{\omega}$ be the minimal concave majorant of $\omega$. Then $\lim _{t \rightarrow \infty} \frac{\omega(t)}{t}=\lim _{t \rightarrow \infty} \frac{\bar{\omega}(t)}{t}<\infty$ and $\omega^{\prime}(0)=\bar{\omega}^{\prime}(0)$.

Proof. Note that $\frac{\bar{\omega}(t)}{t}$ is a nonincreasing function on $(0, \infty)$ since $\bar{\omega}$ is concave. This ensures the existence of $\lim _{t \rightarrow \infty} \frac{\bar{\omega}(t)}{t}$. The proof of Lemma B in [2] contains the inequality

$$
\bar{\omega}(t)<\frac{\omega\left(t_{0}\right)}{t_{0}} t+\omega\left(t_{0}\right), \text { for } t>0 \text { and } t_{0}>0 .
$$

Hence $\lim _{t \rightarrow \infty} \frac{\bar{\omega}(t)}{t} \leq \frac{\omega\left(t_{0}\right)}{t_{0}} \leq \frac{\bar{\omega}\left(t_{0}\right)}{t_{0}}$ and the first statement of the lemma follows.

Since $\bar{\omega}$ is concave, it has a finite or infinite derivative from the right $\bar{\omega}^{\prime}(0)$. By passing to the limit as $t_{0} \rightarrow 0^{+}$in $(1)$ we get $\bar{\omega}(t) \leq t \lim _{t \rightarrow 0} \inf \frac{\omega(t)}{t}$; whence $\bar{\omega}^{\prime}(0) \leq \lim _{t \rightarrow 0} \inf \frac{\omega(t)}{t}$. On the other hand, $\lim _{t \rightarrow 0} \sup \frac{\omega(t)}{t} \leq \bar{\omega}^{\prime}(0)$. So $\omega^{\prime}(0)=\bar{\omega}^{\prime}(0)$.

Below we make use of the following smoothing method [3]. For every $\delta>0$ let $\varphi(\delta, t)$ be an even infinitely differentiable function on $(-\infty, \infty)$ with $\varphi(\delta, t)>0$ on $(-\delta, \delta)$ and $\varphi(\delta, t)=0$ elsewhere. Assume also $\int_{-\delta}^{\delta} \varphi(\delta, t) d t=1$. 
Then given a continuous function $f$ on $(-\infty, \infty)$ the function of $t$ defined by

$$
f(\delta, t)=\int_{-\infty}^{\infty} f(x) \varphi(\delta, x-t) d x=\int_{-\delta}^{\delta} f(x+t) \varphi(\delta, x) d x
$$

is infinitely differentiable and $\lim _{\delta \rightarrow 0} f(\delta, t)=f(t)$ uniformly over any compact set in $(-\infty, \infty)[3$, p. 46]. It is easy to show that if $f$ is concave, then so is $f(\delta, t)$.

Actually, this method will be applied to special functions defined on a finite interval. Specifically, let $f$ be a continuous function defined on $[p, q]$ so that $f$ is affine on $\left[p, p_{1}\right]$ and $\left[q_{1}, q\right]$ with different slopes, $p<p_{1}<q_{1}<q$. Further without special mention $f$ is extended to $(-\infty, \infty)$ so that the resulting function is affine on $\left(-\infty, p_{1}\right]$ and $\left[q_{1}, \infty\right)$ respectively. For convenience the extension of $f$ is also denoted by $f$. It is easy to check that such a function satisfies $f(\delta, t)=f(t)$ outside $\left(p_{1}-\delta, q_{1}+\delta\right)$. We will adhere to the following convention. If $L$ is the tangent at a point $(x, y)$ to the graph of some function, then we say simply that $L$ is the tangent to the graph at the point $x$. The topology on any interval is induced, as usual, by that on $(-\infty, \infty)$.

Lemma 2. Let $y=k_{1} t+d_{1}$ and $y=k_{2} t+d_{2}$ be the tangents to the graph of a concave function $v$ at points $a<b$ respectively. Suppose that these tangents meet outside the graph of $v$. Then given any $\varepsilon>0$ there exists an infinitely differentiable concave function $u$ on $[a, b]$ with the following properties:

1. $0 \leq u(t)-v(t)<\varepsilon$ for all $t \in[a, b]$;

2. $u(t)=k_{1} t+d_{1}$ on some neighborhood of a and $u(t)=k_{2} t+d_{2}$ on some neighborhood of $b$.

Proof. Denote by $\left(c, y_{0}\right)$ the point of intersection of the two tangents. Clearly $k_{1}>k_{2}, a<c<b$ and $y_{0}>v(c)$. The function $v_{1}$ defined by $v_{1}(t)=$ $k_{1} t+d_{1}$ on $[a, c]$ and $v_{1}(t)=k_{2} t+d_{2}$ on $[c, b]$ is concave. Let $\varepsilon>0$. Set $v_{0}(t)=\min \left\{v_{1}(t), v(t)+\frac{\varepsilon}{2}\right\}, a \leq t \leq b$. Then $0 \leq v_{0}(t)-v(t) \leq \frac{\varepsilon}{2}$. If $t=a$ or $t=b$, then $v_{0}(t)-v(t)=0$, while $v_{0}(c)-v(c)>0$. Consequently, there are an $\varepsilon_{0}>0$ and points $a_{1} \in(a, c), b_{1} \in(c, b)$ such that $\varepsilon_{0}<\frac{\varepsilon}{2}$ and $v_{0}\left(a_{1}\right)-v\left(a_{1}\right)=v_{0}\left(b_{1}\right)-v\left(b_{1}\right)=\varepsilon_{0}$. The definition of $v_{0}$ and the concavity of $v$ imply $v_{0}(t)=v_{1}(t)$ for $t \in\left[a, a_{1}\right] \cup\left[b_{1}, b\right]$ and $v_{0}(t)-v(t) \geq \varepsilon_{0}$ for $a_{1} \leq t \leq b_{1}$. By the continuity of $v_{0}$ and $v$ we have also $v_{0}(t)-v(t) \geq \frac{1}{2} \varepsilon_{0}$ for $t \in\left[a_{1}-\delta, b_{1}+\delta\right]$ if $\delta$ is sufficiently small, $\delta>0$, provided $a_{1}-\delta>a$ and $b_{1}+\delta<b$.

Let us smooth $v_{0}$ by means of the above method. For a sufficiently small $\delta$ we get an infinitely differentiable concave function $u(t)=v_{0}(\delta, t)$ with the 
following properties. The inequality $\left|u(t)-v_{0}(t)\right|<\frac{1}{2} \varepsilon_{0}$ holds for all $t \in[a, b]$ and hence $u(t)-v(t)<\varepsilon$. If $t \in\left[a, a_{1}-\delta\right] \cup\left[b_{1}+\delta, b\right]$, then $u(t)=v_{1}(t) \geq v(t)$. If $t \in\left[a_{1}-\delta, b_{1}+\delta\right]$, then $u(t)-v(t)=u(t)-v_{0}(t)+v_{0}(t)-v_{1}(t)>-\frac{1}{2} \varepsilon_{0}+\frac{1}{2} \varepsilon_{0}=0$, completing the proof.

Proof of the Theorem. Observe that $\omega(\mu t) \leq \omega_{0}(\mu t)<(1+\mu) \omega(t)$ for all $t>0$ and $\mu \in I$ if and only if $\omega(t) \leq \omega_{0}(t)<(1+\mu) \omega\left(\frac{t}{\mu}\right)$ for all $t>0$ and $\mu \in I$. Let $\bar{\omega}$ be the minimal concave majorant of $\omega$ and let $I=\left[\mu_{1}, \mu_{2}\right]$. First we construct $\omega_{0}$ sometimes disregarding the statement concerning $\omega_{0}(t)=\omega^{\prime}(0) t$. Let us consider different cases. The case with a linear $\bar{\omega}$ is trivial. Assume that $\bar{\omega}$ is of the form: $\bar{\omega}(t)=k_{1} t$ on $\left[0, t_{0}\right]$ and $\bar{\omega}=k_{2} t+d$ on $\left[t_{0}, \infty\right)$ with $k_{1}>k_{2}$ and some $t_{0}>0$. The minimality of $\bar{\omega}$ implies $\bar{\omega}\left(t_{0}\right)=\omega\left(t_{0}\right)$. If $k_{2}=0$, then $\omega(t)=\bar{\omega}(t)=d$ on $\left[t_{0}, \infty\right)$. Therefore $(1+\mu) \omega\left(\frac{t}{\mu}\right)-\bar{\omega}(t)=\mu d \geq \mu_{1} d$ for all $\mu \in I$ and sufficiently large $t$. If $k_{2}>0$, then we argue as follows. By Lemma $1, \lim _{t \rightarrow \infty} \frac{\omega(t)}{t}=\lim _{t \rightarrow \infty} \frac{\bar{\omega}(t)}{t}=k_{2}$. Then given any $\varepsilon>0$ there is an arbitrarily large $\tau>0$ such that $\frac{1}{t} \bar{\omega}(t) \leq(1+\varepsilon) k_{2}$ and $\frac{\mu}{t} \omega\left(\frac{t}{\mu}\right) \geq(1-\varepsilon) k_{2}$ for $t \geq \tau$ and all $\mu \in I$. If $\varepsilon$ is sufficiently small, then a simple calculation shows that $(1+\mu) \omega\left(\frac{t}{\mu}\right)-\bar{\omega}(t) \geq \frac{1}{2} k_{2} \tau \mu_{2}^{-1}$, where $t \geq \tau, \mu \in I$. Thus in both cases: $k_{2}=0$ and $k_{2}>0$, we have $\min _{t \geq t_{0}, \mu \in I}\left((1+\mu) \omega\left(\frac{t}{\mu}\right)-\bar{\omega}(t)\right)=2 m$ with $m>0$. Denote by $\left(t_{1}, y_{1}\right)$ the point of intersection of the lines $y=k_{1} t$ and $y=k_{2} t+d+m$. Set $\omega_{1}(t)=k_{1} t$ for $0 \leq t \leq t_{1}$ and $\omega_{1}(t)=k_{2} t+d+m$ for $t \geq t_{1}$. Then $\omega_{1}\left(t_{1}\right)>\bar{\omega}\left(t_{1}\right)$ and $\bar{\omega}(t) \leq \omega_{1}(t)<(1+\mu) \omega\left(\frac{t}{\mu}\right)$ for all $t>0$. By smoothing we obtain a function $\omega_{0}(t)=\omega_{1}(\delta, t)$ with all the desired properties if $\delta$ is sufficiently small.

Consider the case, where $\bar{\omega}(t)=k_{1} t$ on $\left[0, a_{1}\right]$ and $\bar{\omega}(t)=k_{2} t+d$ on $\left[b_{1}, \infty\right)$ with $0<a_{1}<b_{1}$ provided the lines $y=k_{1} t$ and $y=k_{2} t+d$ meet outside the graph of $\bar{\omega}$. In this case the conclusion of the theorem follows at once from Lemma 2.

Assume now that $\bar{\omega}(t)=k t$ on some interval $\left[0, b_{1}\right]$, but $\bar{\omega}$ is not affine on any infinite interval. We suppose $\bar{\omega}^{\prime}\left(b_{1}\right)=k$, since otherwise $b_{1}$ can be replaced by a smaller positive number. Choose a $b_{2}>b_{1}+1$ so that $\bar{\omega}^{\prime}\left(b_{2}\right)$ exists and the tangents to the graph of $\bar{\omega}$ at $b_{1}$ and $b_{2}$ meet outside the graph. Such a choice is possible, since otherwise $\bar{\omega}$ would be affine on some infinite interval. Again, choose a $b_{3}>b_{2}+1$ so that $\bar{\omega}^{\prime}\left(b_{3}\right)$ exists and the tangents at $b_{2}$ and $b_{3}$ meet outside the graph of $\bar{\omega}$. Continuing this process we obtain a sequence $\left\{b_{i}\right\}_{i=1}^{\infty}$ such that $b_{i+1}>b_{i}+1$ and the tangents at $b_{i}$ and $b_{i+1}$ meet outside the graph of $\bar{\omega}$. Denote by $L_{i}$ the tangent at $b_{i}, i \in \mathbb{N}$. Set $\varepsilon_{i}=\min _{b_{i} \leq t \leq b_{i+1}, \mu \in I}\left((1+\mu) \omega\left(\frac{t}{\mu}\right)-\bar{\omega}(t)\right)$. By Lemma 2 for each $i \in \mathbb{N}$ there is infinitely differentiable concave function $\omega_{i 0}$ on $\left[b_{i}, b_{i+1}\right]$ such that $0 \leq \omega_{i 0}(t)-$ 
$\bar{\omega}(t)<\varepsilon_{i}$ and hence $\omega(t) \leq \omega_{i 0}(t)<(1+\mu) \omega\left(\frac{t}{\mu}\right), t \in\left[b_{i}, b_{i+1}\right], \mu \in I$. Moreover, the graph of $\omega_{i 0}$ coincides with $L_{i}$ on some neighborhood of $b_{i}$ and with $L_{i+1}$ on some neighborhood of $b_{i+1}$. Set $\omega_{0}(t)=k t$ on $\left[0, b_{1}\right]$ and $\omega_{0}(t)=\omega_{i 0}(t)$ on $\left[b_{i}, b_{i+1}\right], i \in \mathbb{N}$. The values of $\omega_{i 0}$ on consecutive intervals are coordinated so that $\omega_{0}$ is infinitely differentiable. The concavity of $\omega_{0}$ is obvious. Thus the conclusion of the theorem holds.

Consider the case where $\bar{\omega}(t)=k t+d$ on some half-line $\left[a_{1}, \infty\right), a_{1}>0$, and $\bar{\omega}$ is not linear on any neighborhood of zero. We can assume that $\bar{\omega}^{\prime}\left(a_{1}\right)=k$. As in the preceding case we choose a decreasing sequence $\left\{a_{i}\right\}_{i=1}^{\infty}$ so that $a_{i} \rightarrow 0$ as $i \rightarrow \infty$ and the tangents at $a_{i}, a_{i+1}$ meet outside the graph of $\bar{\omega}$. Applying again Lemma 2 and setting $\omega_{0}(0)=0$ we construct a function $\omega_{0}$ so that $\omega_{0}$ has the desired properties except for the last statement of the theorem.

Suppose that $\bar{\omega}$ is not affine on $\left[t_{1}, \infty\right)$ as well as on $\left[0, t_{2}\right]$ for any positive $t_{1}, t_{2}$. Choose an $a_{1}>0$ so that $\bar{\omega}^{\prime}\left(a_{1}\right)$ exists and set $b_{1}=a_{1}$. We determine two sequences $\left\{a_{i}\right\}_{i=1}^{\infty}$ and $\left\{b_{i}\right\}_{i=1}^{\infty}$ in the same way as in the two previous cases. The further construction being clear we omit the details.

We have still to prove the last statement of the theorem when $\omega^{\prime}(0)<\infty$ and $\bar{\omega}$ is not linear on any neighborhood of zero. To this end let us change our construction somewhat. Choose $\varepsilon>0$ so that $\left(1+\mu_{2}^{-1}\right)(1-\varepsilon)>1$. By Lemma $1 \omega(t) \sim \bar{\omega}^{\prime}(0) t$ as $t \rightarrow 0$. Then for all $\mu \in I$ and $t$ sufficiently small

$$
(1+\mu) \omega\left(\frac{t}{\mu}\right) \geq \frac{1+\mu}{\mu}(1-\varepsilon) \bar{\omega}^{\prime}(0) t \geq\left(1+\mu_{2}^{-1}\right)(1-\varepsilon) \bar{\omega}^{\prime}(0) t>\bar{\omega}^{\prime}(0) t .
$$

Therefore, while constructing the sequence $\left\{a_{i}\right\}_{i=1}^{\infty}$ one can single out a $j$ such that $\bar{\omega}^{\prime}(0) t<(1+\mu) \omega\left(\frac{t}{\mu}\right)$ for $t \in\left(0, a_{j}\right]$ and $\mu \in I$. We define $\omega_{0}$ on $\left[a_{j}, \infty\right)$ just as above. If $0<t<a_{j}$, then we construct $\omega_{0}$ in a different way. Let $B$ denote the point $\left(a_{j}, \bar{\omega}\left(a_{j}\right)\right)$. The tangent at $B$ to the graph of $\bar{\omega}$ meets the line $y=\bar{\omega}^{\prime}(0) t$ at a point $A$ with an abscissa $a \in\left(0, a_{j}\right)$. The union of the line segments $O A$ and $A B$ is the graph of some function $\omega_{2}$ on $\left[0, a_{j}\right]$. The segment $A B$, except for $A$, lies under the half-line $O A$. It follows that $\omega_{2}(t)<(1+\mu) \omega\left(\frac{t}{\mu}\right)$ for $t \in\left(0, a_{j}\right]$ and $\mu \in I$. Observe also that $\omega_{2}(a)>\bar{\omega}(a)$. Smoothing $\omega_{2}$ with a sufficiently small $\delta>0, \delta<\min \left\{a, a_{j}-a\right\}$, and setting $\omega_{0}(t)=\omega_{2}(\delta, t)$ on $\left[0, a_{j}\right]$ we finally obtain $\omega_{0}$ on $[0, \infty)$ with all the properties claimed in the theorem.

Clearly, the unimprovability asserted in Lemma B remains valid for $\omega_{0}$.

Let us remark that the condition of the theorem cannot be weakened by assuming only $\mu \in(0, \infty)$ instead of $\mu \in I$. Indeed, take $\omega$ such that $\omega(t)=t$ on $[0,1]$ and $\omega(t)=1$ on $[1, \infty)$. Then $\omega_{0}(t)=\omega(t)$ for $0 \leq t \leq 1$, since otherwise $\omega_{0}^{\prime}(0) \neq \omega^{\prime}(0)$. In order that $\omega_{0}$ be a concave differentiable majorant of $\omega$ it is necessary that $\omega_{0}(t)>\omega(t)$ for $t>1$ and, in particular, $\omega_{0}(2)>1$. 
If $\omega_{0}(2)<(1+\mu) \omega\left(\frac{2}{\mu}\right)$ for all $\mu>0$, then taking the limit as $\mu \rightarrow 0$ we obtain $\omega_{0}(2) \leq 1$ contradicting $\omega_{0}(2)>1$. It remains unclear if the condition $\mu_{1} \leq \mu \leq \mu_{2}$ can be weakened by assuming only $\mu \geq \mu_{1}>0$.

Lemma A does not answer the question if the factor 2 can be replaced by a smaller value depending on $\omega$. Lemma $\mathrm{B}$ gives rise to a similar question with any $\mu>0$. It is also noteworthy that $\bar{\omega}(\mu t)<(1+\mu) \omega(t)$ if $\omega$ is defined on a finite interval $[0, l]$, since $\omega$ can be extended to $[0, \infty)$ by setting $\omega(t)=\omega(l)$ for $t>l$. However, in this case the unimprovability of the indicated inequality requires a complementary study. Indeed, taking $\mu=1$ and $t=l$ we can write $\bar{\omega}(l)=\omega(l)$ instead of $\bar{\omega}(l)<2 \omega(l)$. Below we construct an example which shows the unimprovability of $\bar{\omega}(\mu t)<(1+\mu) \omega(t)$ in a sense different from that in Lemma B and thereby we complement our theorem. Incidentally, the same example enables us to remove in Lemma $\mathrm{B}$ the restriction that $\mu$ be a natural number.

Take a sequence of positive numbers $\left\{q_{m}\right\}_{m=-\infty}^{\infty}$ so that $q_{m} \rightarrow \infty$ as $|m| \rightarrow \infty$. Let $a_{0}=c_{0}=1$. For each integer $m$ we determine inductively a triple $a_{m}, b_{m}, a_{m+1}$ that forms a geometric progression with $q_{m}$ as the ratio. Applying again induction on $m$ we define a continuous nondecreasing function $\omega$ on $[0, \infty)$ as follows. $\omega(0)=0, \omega(t)=c_{m}$ for $a_{m} \leq t \leq b_{m}$ and $\omega(t)=\frac{c_{m}}{b_{m}} t$ for $b_{m} \leq t \leq a_{m+1}$ with suitable constants $c_{m}$. Note the following property of the graph of $\omega$. Given any $x>0$, the points of the chord joining $(0,0)$ and $(x, \omega(x))$ lie under or on the graph. It follows that $\omega$ is semiadditive. Thus, $\omega$ is a modulus of continuity.

Let $\omega_{l}$ denote the restriction of $\omega$ to the finite interval $[0, l]$ and let $\varphi_{l}$ be a concave majorant of $\omega_{l}$. Fix a $\mu>0$. Consider only those integers $m$ that satisfy $a_{m+1} \leq l$ and $\mu b_{m} \in\left[a_{m}, a_{m+1}\right]$. The points $\left(a_{m}, c_{m}\right)$ and $\left(a_{m+1}, c_{m+1}\right)$ belong to the graph of $\omega_{l}$. It is not hard to check that the line segment joining these points is described by $y=\frac{c_{m}}{q_{m}+1}\left(\frac{t}{a_{m}}+q_{m}\right), a_{m} \leq t \leq a_{m+1}$. If $t=$ $\mu b_{m}$, then $y=\frac{(1+\mu) q_{m}}{q_{m}+1} \omega_{l}\left(b_{m}\right)$. It follows that $\varphi_{l}\left(\mu b_{m}\right) \geq \frac{(1+\mu) q_{m}}{q_{m}+1} \omega_{l}\left(b_{m}\right)$ since $\varphi_{l}$ is a concave majorant of $\omega_{l}$. The same argument is valid for any concave majorant $\varphi$ of $\omega$ on $[0, \infty)$. Clearly $\frac{(1+\mu) q_{m}}{q_{m}+1} \rightarrow 1+\mu$ as $|m| \rightarrow \infty$. Thus, there is a single modulus of continuity $\omega$ such that any factor $1+\mu$ in $\bar{\omega}(\mu t)<(1+\mu) \omega(t)$ cannot be reduced, no matter if we consider functions on a finite or infinite interval.

The function $\omega$ constructed above can be used in another way. Let $\mu>0$ and $t_{0}>0$ be given. For any integer $m$ set $\omega(t, m)=\omega\left(\frac{b_{m}}{t_{0}} t\right), t \geq 0$. If $\psi(t)$ is concave majorant of $\omega(t, m)$, then $\varphi(t)=\psi\left(\frac{t_{0}}{b_{m}} t\right)$ is a concave majorant of 
$\omega(t)$. Therefore $\psi\left(\mu t_{0}\right) \geq \frac{(1+\mu) q_{m}}{q_{m}+1} \omega\left(t_{0}, m\right)$. Since $q_{m}$ is arbitrarily large, it follows that the last statement of Lemma B remains valid with any $\mu>0$.

Acknowledgment

The author is grateful to P. L. Ul'janov for the setting of the problem and his advice.

\section{References}

[1] A. V. Efimov, Linear methods of approximating continuous periodic functions, Math. Sb., 54 (1961), 51-90. (Russian)

[2] N. P. Korneychuk, On the precise constant in Jackson's inequality for continuous periodic functions, Math. Zametki, 32 (1982), 669-674. (Russian)

[3] M. W. Hirch, Differential topology, Sprynger-Verlag, New York, etc., 1976. 
A. V. Medvedev 